sleep and were less physically active. Metabolic syndrome prevalence was $33.1 \%$ among the participants. The adjusted odds ratio for the shift workers to develop metabolic syndrome was 0.55 (95\% CI 0.24-1.29) with a P value of 0.17 . Conclusion Metabolic syndrome was present in every third person among the study participants and there was no significant association with shift work.

\section{Poster Presentation}

\section{Intervention Studies}

\section{A LITERATURE REVIEW OF WORKPLACE INTERVENTIONS WITH RESPECT TO RISK MANAGEMENT MEASURES AND THEIR IMPACT ON OCCUPATIONAL EXPOSURE LEVELS TO HAZARDOUS SUBSTANCES}

Susann Wothe. Federal Institute for Occupational Safety and Health $(B A \mu A)$, Unit 4.1 Exposure Scenarios, Dortmund, North Rhine-Westphalia, Germany

\subsection{6/oemed-2017-104636.45}

Background and aims: Workplace intervention studies play an important role in supporting and complementing scientific validation of non-intervention assessments of the effectiveness of risk management measures (RMMs) under controlled conditions. We are reviewing a collection of published workplace intervention studies with particular focus on studies assessing changes in occupational exposure to hazardous substances with a broad scope spanning a variety of approaches in different industries.

Methods Workplace interventions were defined as events aimed at reducing occupational exposure to hazardous substances at the workplace or where reductions occurred as a side effect, e.g. due to changes in the production process. Intervention studies published in English from 1999 up to January 2017 were considered for inclusion based on a systematic search of Pubmed.

Results In total 50 intervention studies have been included in this review including, but not limited to, studies in the metal industry (10), hospitals (4), bakeries (3), on welding (6), or dust in construction (4). Overall the interventions reviewed have succeeded at reducing exposure levels.

Conclusion There is evidence that decreases in workplace exposure levels to hazardous substances followed a variety of workplace interventions in a variety of industries underlining the benefits of implementing RMMs at workplaces. However, a direct comparison of a specific RMM among different studies, even when focussing on one specific industry, remains difficult as the majority of studies assessed a set of different RMMs; hence the quantification of the impact of individual interventions on exposure remains difficult due to the heterogeneity in methods.

\section{Oral Presentation}

Shift Work

\section{OBJECTIVELY MEASURED NON-OCCUPATIONAL AND OCCUPATIONAL PHYSICAL ACTIVITY LEVELS OF SHIFT WORKERS COMPARED TO NON-SHIFT WORKERS}

\begin{abstract}
${ }^{1,2}$ Bette Loef*, ${ }^{3}$ Debbie van Baarle, ${ }^{2}$ Allard van der Beek, ${ }^{4}$ Andreas Holtermann, ${ }^{1}$ Karin Proper. ${ }^{1}$ Center for Nutrition, Prevention and Health Services, National Institute for Public Health and the Environment, Bilthoven, The Netherlands; ${ }^{2}$ Department of Public and Occupational Health, Amsterdam Public Health research institute, VU University Medical Centre, Amsterdam, The Netherlands; ${ }^{3}$ Center for Immunology of Infectious Diseases and Vaccines, National Institute for Public Health and the Environment, Bilthoven, The Netherlands; ${ }^{4}$ National Research Centre for the Working Environment, Copenhagen, Denmark
\end{abstract}

\subsection{6/oemed-2017-104636.46}

Background Shift work may alter workers' physical activity (PA) level, making PA a potential underlying mechanism of the negative health effects of shift work. As prior studies on shift work and PA have generally used self-reported, overall PA measures, the results may be susceptible to bias. Therefore, our aim was to compare objectively measured non-occupational and occupational PA levels between shift workers and non-shift workers.

Methods Data were used from Klokwerk+, a prospective cohort study examining the health effects of shift work among health care workers. In total, 401 rotating and/or night shift workers and 78 non-shift workers were included, who wore Actigraph GT3X+ accelerometers for 7 consecutive days. Time spent sitting, standing, walking, running, stairclimbing, and cycling during leisure and at work was estimated using Acti4-software. Linear regression was used to compare proportions of time spent in these activities between shift and nonshift workers.

Results Average accelerometer wear-time was 105.9 hours $(\mathrm{SD}=14.0)$ over an average of 6.9 days $(\mathrm{SD}=0.6)$. No differences between shift workers and non-shift workers were found in PA behaviours during leisure-time $(\mathrm{p}>0.05)$. At work, shift workers were less sedentary $(B=-10.6 \quad$ (95\%-CI $=-14.3$ $-6.8)$ ) and spent larger proportions of the time standing $(\mathrm{B}=9.5 \quad(95 \%-\mathrm{CI}=6.4-12.6)) \quad$ and walking $\quad(\mathrm{B}=1.2 \quad(95 \%-$ $\mathrm{CI}=0.1-2.2))$ than non-shift workers.

Conclusions Non-occupational PA levels of shift workers were similar to that of non-shift workers, but shift workers were more physically active (i.e. standing/walking) at work. Future research should focus on the role of this difference in occupational PA in the health effects of shift work. 